Neues Archiv

für

sächsische Geschichte 


\section{Neues Archiv für \\ sächsische Geschichte}

69. Band $\cdot 1998$

Herausgegeben

von

Karlheinz Blaschke

1999

VERLAG HERMANN BÖHLAUS NACHFOLGER WEIMAR 
Manuskripte und Rezensionsexemplare

werden an den Herausgeber erbeten: Professor Dr. Karlheinz Blaschke,

Am Park, 01468 Friedewald

Redaktion: Uwe John

Die Deutsche Bibliothek - CIP-Einheitsaufnahme

Neues Archiv für sächsische Geschichte. - Weimar:

Verlag Hermann Böhlaus Nachfolger Weimar.

Erscheint jährlich. - Von 1943 bis 1992 nicht erschienen.

- Aufnahme nach Bd. 64 (1993)

ISSN 0944-8195

Bd. 64. 1993. $-(1994)-$

ISBN 978-3-7400-0865-9

ISBN 978-3-476-02964-5 (eBook)

DOI 10.1007/978-3-476-02964-5

ISSN 0944-8195

Erschienen im Verlag Hermann Böhlaus Nachfolger Weimar GmbH \& Co.

(C) 1999 Springer-Verlag GmbII Deutschland

Ursprünglich erschienen bei Verlag I Iermann Böhlaus Nachfolger Weimar 1999

Alle Rechte vorbehalten. Ohne schriftliche Genehmigung des Verlages ist es nicht gestattet, das Werk unter Verwendung mechanischer und anderer Systeme in irgendeiner Weise zu verarbeiten und zu verbreiten. Insbesondere vorbehalten sind die Rechte der Vervielfältigung - auch von Teilen des Werkes - auf photomechanischem oder ähnlichem Wege, der tontechnischen Wiedergabe, der Vortrags, der Funk- und Fernsehsendung, der Speicherung in Datenverarbeitungsanlagen, der Übersetzung und der literarischen oder anderweitigen Bearbeitung. 


\section{Inhalt}

Thomas Vogtherr, Thomas Ludwig, Die Äbtereihe des Benediktinerklosters St. Jakob in Pegau

Wieland Held, Christoph von Carlowitz. Ein sächsischer Adliger des 16. Jahrhunderts zwischen diplomatischer Tätigkeit und unternehmerischem Engagement.

Marcus Ventzke, Zwischen Kaisertreue und Interessenpolitik. Sachsen-Altenburg zu Beginn des 17. Jahrhunderts.

Detlef Döring, Abraham von Sebottendorf. Ein kursächsischer Politiker aus der Zeit des Dreißigjährigen Krieges in seinen Briefen an Reinhard Rose (mit Quellenanhang)

Markus A. Denzel,»Universal Regeln der Leipiger Wechsel Negotien«. Ein kaufmännisches Rechenbuch von Christlieb von Clausberg (um 1730)

Katharina Middell, Leipziger Sozietäten im 18. Jahrhundert. Die Bedeutung der Soziabilität für die kulturelle Integration von Minderheiten

Gunda Ulbricht, Die verfassungsrechtliche Trennung von Stadt und Landgemeinde. Ein zentrales Problem der sächsischen Kommunalgesetzgebung im 19. Jahrhundert

Peter Kunze, Eine sorbische Petition zur Sprachenpolitik 1848/49..

Thomas Klein, Wahlprüfungen zu den Reichstagswahlen im Königreich Sachsen 1867-1918 (Schluß) 
Forschung und Diskussion

Eckhart Leisering, Die Rechte der Wettiner als Reichsfürsten. Eine Aufzeichnung von 1350

Henning Steinfübrer, Die Edition der ältesten erhaltenen Leipziger Ratsbücher (1466-1500). Ein aktuelles Forschungsprojekt zur sächsischen Städtegeschichte im Spätmittelalter

Ute Essegern, Elisabeth von Braunschweig-Wolfenbüttel. Eine Fürstin am Dresdner Hof...

Karlheinz Blaschke, Landesgeschichte ohne geschichtliches Land was ist Landesgeschichte? Bemerkungen zu zwei Veröffentlichungen über die Geschichte des Landes Sachsen-Anhalt

\section{Rezensionen}

Sächsische Bibliographie. Berichtsjahr 1996 und Nachträge (M. Kobuch) ......

Landesgeschichte als Herausforderung und Programm. Karlheinz Blaschke zum 70. Geburtstag, hrsg. von U. John und J. Matzerath (J. John)

Landesgeschichte in Sachsen. Tradition und Innovation, hrsg. von $R$. Aurig, S. Herzog und S. Lässig (H. Heller)

Recht und Rechtswissenschaft im mitteldeutschen Raum. Symposion für Rolf Lieberwirth anläßlich seines 75. Geburtstags, hrsg. von H. Lück (A. Laufs) ..

Vom rechten Maß der Dinge. Beiträge zur Wirtschafts- und Sozialgeschichte. Festschrift für Harald Witthöft zum 65. Geburtstag (M. Straube)

Deutsche Königspfalzen. Beiträge zu ihrer historischen und archäologischen Erforschung. Vierter Band: Pfalzen - Reichsgut - Königshöfe, hrsg. von L. Fenske (H. K. Schulze)

H. Assing, Brandenburg, Anhalt und Thüringen im Mittelalter. Askanier und Ludowinger beim Aufbau fürstlicher Territorialherrschaften. Zum 65. Geburtstag des Autors hrsg. von T. Köhn, L. Partenheimer, U. Zietmann (P. Neumeister)

Chronik vom Petersberg (Cronica Montis Sereni) nebst der Genealogie der Wettiner (Genealogia Wettinensis), übers. und erl. von W. Kirsch (S. Pätzold) ..

„Raubritter“ oder „Rechtschaffene vom Adel“? Aspekte von Politik, Friede und Recht im späten Mittelalter, hrsg. von K. Andermann (J. Schneider) 
M. Müller, Die spätmittelalterliche Bistumsgeschichtsschreibung. Überlieferung und Entwicklung (S. Pätzold)

H. Lück, Die kursächsische Gerichtsverfassung 1423-1550 (B. Diestelkamp)...

W. Buchbolz, Geschichte der öffentlichen Finanzen in Europa in Spätmittelalter und Neuzeit (U. Schirmer)

Geschichte des sächsischen Adels, hrsg. von K. Keller und J. Matzerath in Zusammenarbeit mit Chr. Klecker und K.-D. Wintermann (R. Endres)

Der Herrscher in der Doppelpflicht. Europäische Fürsten und ihre beiden Throne, hrsg. von H. Duchbardt (J. Bruning)

Ständefreiheit und Staatsgestaltung in Ostmitteleuropa. Übernationale Gemeinsamkeiten in der politischen Kultur vom 16.-18. Jahrhundert, hrsg. von J. Bablcke, H.-J. Bömelburg und N. Kersken ( M. Köhler-Baur).

Die Territorien des Reiches im Zeitalter der Reformation und Konfessionalisierung. Heft 7: Bilanz - Forschungsperspektiven - Register, hrsg. von A. Schindling und W. Ziegler ( S. Bräuer).

Martin Luther und seine Universität. Vorträge anläßlich des 450. Todestages des Reformators, hrsg. von $H$. Lück (D. Stievermann)

H. Scheible, Melanchthon und die Reformation. Forschungsbeiträge, hrsg. von G. May und R. Decot (K. Blaschke)

W. Held, 1547. Die Schlacht bei Mühlberg/Elbe. Entscheidung auf dem Wege zum albertinischen Kurfürstentum Sachsen (J. Mötsch)

P. Gabriel, Fürst Georg III. von Anhalt als evangelischer Bischof von Merseburg und Thüringen 1544-1548/50 (J. Herrmann)

U. Gittel, Die Aktivitäten des Niedersächsischen Reichskreises in den Sektoren „Friedenssicherung" und „Policey“ (1555-1682) (Th. Nicklas).

F. Müller, Kursachsen und der Böhmische Aufstand 1618-1622 (K. Blaschke) .

H. Duchhardt, Balance of Power und Pentarchie 1700-1785 (R. Pommerin).

$K$. Heldt, Der vollkommene Regent. Studien zur panegyrischen Casuallyrik am Beispiel des Dresdner Hofes Augusts des Starken (R. Jacobsen).

H. Kiesewetter, Das einzigartige Europa. Zufällige und notwendige Faktoren der Industrialisierung (K. Blaschke)

G. G. Iggers, Deutsche Geschichtswissenschaft. Eine Kritik der traditionellen Geschichtsauffassung von Herder bis zur Gegenwart (K. Blaschke)

K. Rudolph/I. Weuster, Bibliographie zur Geschichte der Demokratiebewegung in Mitteldeutschland (1789-1933) (J. John) 
Bürgerkultur im 19. Jahrhundert. Bildung, Kunst und Lebenswelt; [Lothar Gall zum 60. Geburtstag], hrsg. von D. Hein und A. Schulz (S. Taschka)

Unternehmer und technischer Fortschritt, hrsg. von F. Schinzinger (Th. Hänseroth)

H. Zwabr, Revolutionen in Sachsen. Beiträge zur Sozial- und Kulturgeschichte (D. J. Weiß)

F. Zimmer, Bismarcks Kampf gegen Kaiser Franz Joseph. Königgrätz und seine Folgen (K. Blaschke)

Der Kulturkampf, hrsg. von $R$. Lill (W. Halder)

Geschichtsforschung in Sachsen. Von der Sächsischen Kommission für Geschichte zur Historischen Kommission bei der Sächsischen Akademie der Wissenschaften zu Leipzig, 1896-1996 (S. Bräuer)

F. Webrmeister, Fortbildungsschule in Sachsen I. Allgemeine und gewerbliche Fortbildungsschule in Sachsen im Spannungsfeld schulpolitischer und gewerblicher Interessen (1815-1933) (H. Voit)

K. Wehrmeister, Fortbildungsschule in Sachsen II. Geschlechtsspezifische Erziehung und berufliche Qualifikation in der sächsischen Mädchenfortbildungsschule (1835-1925) (H. Voit)

J. Frotscher, Volksschullehrerausbildung in Dresden 1923-1931 (F. Wehrmeister)

M. Schmeitzner/M. Rudloff, Geschichte der Sozialdemokratie im Sächsischen Landtag. Darstellung und Dokumentation 1877-1997 (K. Blaschke) .

B. Lapp, Revolution from the Right. Politics, Class, and the Rise of Nazism in Saxony, 1919-1933 (R. Pommerin)

Dresden unterm Hakenkreuz, hrsg. von R. Pommerin (U. von Hehl)

Ines Reich, Carl Friedrich Goerdeler. Ein Oberbürgermeister gegen den NSStaat (G. Wiemers)

Sowjetische Speziallager in Deutschland 1945 bis 1950, Bd. 1: Studien und Berichte, hrsg. von $A$. von Plato (W. Halder)

H. Amos, Justizverwaltung in der SBZ/DDR. Personalpolitik 1945 bis Anfang der 50er Jahre (K. Blaschke)

T. Möblenbrock, Kirche und Bodenreform in der Sowjetischen Besatzungszone Deutschlands 1945-1949 (U. Kluge)

Die Ost-CDU. Beiträge zu ihrer Entstehung und Entwicklung, hrsg. von $M$. Richter und M. Rißmann (R. Baus) 
P. Russig, Wilhelm Grothaus - Dresdner Antifaschist und Aufstandsführer des 17. Juni (P. E. Fäßler)

M. Richter, Die Staatssicherheit im letzten Jahr der DDR (S. Bräuer) ..

50 Jahre Max-Planck-Gesellschaft zur Förderung der Wissenschaften (G. Wiemers)

Zeit und Ewigkeit: 128 Tage in St. Marienstern, Ausstellungskatalog der Ersten Sächsischen Landesausstellung hrsg. von J. Oexle, M. Bauer, M. Winzler (J. Oberste)

Museen in Sachsen (U. John)

Die Dresdner Frauenkirche. Jahrbuch zu ihrer Geschichte und zu ihrem archäologischen Wiederaufbau (U. John)

Quellen zur Geschichte Thüringens. Bd. 6: Feudale Strukturen in der Herrschaft Oppurg vom Ende des Mittelalters bis zum 19. Jahrhundert; Bd. 7: Alltag in der Herrschaft Oppurg vom Ende des Mittelalters bis zum 19. Jahrhundert (M. Schattkowsky)

F. Winkler, Leipzigs Anfänge. Bekanntes - Neues - offene Fragen (G. Billig) ...

D. Peifer, Das religiöse Leipzig oder Buch III des Leipziger Ursprungs und seiner Geschichte (J. Herrmann)

H. A. Nienborg, Description über die Grund-Legung und in richtigen Abriß gebrachte berühmte Handels-Stadt Leipzig Anno 1710; E. Müller, Häuserbuch zum Nienborgschen Atlas (K. Blaschke)

F. Eulenburg, Die Entwicklung der Universität Leipzig in den letzten hundert Jahren. Statistische Untersuchungen. (M. Kobuch)

„Solche Schädlinge gibt es auch in Leipzig!“ Sozialdemokraten und die SED, hrsg. von M. Rudloff und M. Schmeitzner (S. Hoyer)

Chronik des Leipziger Zolls von 1165-1995 (M. Straube)

750 Jahre Kloster St. Marienstern. Festschrift, hrsg. von K. Blaschke, H. Magirius und S. Seifert (J. Oberste)

Kloster Eicha. Wallfahrts-, Antoniter-, Reformations- und Ortsgeschichte, hrsg. von L. Heydick und U. Schirmer (H. Naumann)

1000 Jahre Rochlitz. Festschrift (S. Herzog)

Die Straßennamen der Stadt Rochlitz. Ein Lexikon zur Stadtgeschichte (S. Herzog) 
I. Anders/P. Wolfrum, Görlitz. Historische Ansichten aus vier Jahrhunderten (E.-H. Lemper)

R.Hünecke/U. Hünecke: „Ich, die unterzeichnete Wittwe ...“. Frauen aus Tharandts Geschichte schreiben an die Obrigkeit (G. Ulbricht)

Im Lichte des Halbmonds. Das Abendland und der türkische Orient. Ausstellungskatalog (K. Rudert)

Wort und Name im deutsch-slavischen Kontakt. Ernst Eichler von seinen Schülern und Freunden, hrsg. von K. Hengst, D. Krüger, H. Walther unter Mitarbeit von I. Bily (H. Naumann)

Serbski přisłowny leksikon. Sorbisches Sprichwörterlexikon, hrsg. von S. Hose (L. Udolph)

Autorenverzeichnis 